\title{
The value of performing invasive risk stratification in young patients with the Brugada syndrome
}

\author{
Received: 3 May 2017; Accepted: 6 May 2017; First published online: 19 June 2017
}

The Brugada syndrome is an inherited disease characterised by coved-type ST-segment elevation in the right precordial leads and increased risk for sudden cardiac death. ${ }^{1}$ The phenotypic expression of the disease extends from the completely asymptomatic state to potentially lethal arrhythmias and sudden cardiac death. The disease typically manifests in the fourth decade of life, but severe cases have shown clinical expression during childhood and can result in life-threatening arrhythmias. ${ }^{2,3}$

A provocative test consisting of intravenous administration of a class IC antiarrhythmic drug is a wellestablished tool to unmask the diagnosis of the type I pattern in young patients with suspected Brugada syndrome and a non-diagnostic electrocardiogram.

In contrast, risk stratification by means of ventricular stimulation in this population remains controversial.

The prevalence of the Brugada syndrome is extremely low in the paediatric population $(0.009 \%)^{4}$; moreover, symptoms present in only $30 \%$ of young patients with the Brugada syndrome. Although rarely diagnosed in children, the Brugada syndrome can manifest as sudden cardiac death at a very young age. ${ }^{5}$

There is thus a critical need to accurately identify young patients at increased risk for cardiac arrest, both to minimise mortality and to maintain a low morbidity related to unnecessary device implantation.

Andorin et al recently reported on a multicentric European study that included 106 patients aged $<19$ years. ${ }^{6}$ A baseline electrophysiological study was performed in $22(21 \%)$ patients on the clinical advice of an expert cardiologist, with a positive result in nine (41\%).

Our group has recently reported on the feasibility and outcomes of ventricular stimulation in young patients. ${ }^{3}$ In this series including a cohort of 128 patients $<25$ years, 28 symptomatic patients underwent a ventricular stimulation protocol, and sustained

Correspondence to: M. C. Gonzalez Corcia, MD, Heart Rhythm Management Centre, UZ Brussel-VUB, Laarbeeklaan 101, 1090 Brussels (Belgium); Tel: +32 (0)2 4763038; Fax: +32 (0)2 477 6840; E-mail: mariaceciliagonzalezverbist@gmail.com ventricular fibrillation, or polymorphic ventricular tachycardia was induced in $6(21 \%)$. No ventricular arrhythmias were induced in the 70 ventricular stimulation protocols performed in asymptomatic patients. No complications resulted from the cardiac catheterisation or from the arrhythmia induction.

Performing a single-catheter cardiac catheterisation under propofol sedation has been proven to be a safe procedure when performed by experienced hands and in the appropriate institutional setting, with the provision of advanced life-support facilities. The protocol should include a complete electrophysiological study that provides valuable data on the general electrical performance of the heart. The study should start by measuring the baseline intervals, including the $\mathrm{AH}$ and $\mathrm{HV}$ intervals. The sinus node function should also be evaluated in all patients, by means of sinus node recovery time. The atrioventricular conduction system can be evaluated by measuring the Wenkebach cycle length and the atrioventricular nodal effective refractory period. In patients with either a history of palpitations or with evidence of supraventricular tachycardia from Holter monitoring, an atrial stimulation protocol should be performed. The study concludes with a ventricular stimulation protocol consisting of a maximum of three ventricular extra-stimuli, with a minimum coupling interval of $200 \mathrm{~ms}$, delivered from a single right ventricular site. Results are considered positive in the case of induction of sustained ventricular arrhythmias. A ventricular effective refractory period $<200 \mathrm{~ms}$ and HV interval $>60 \mathrm{~ms}$ are highly suggestive of the Brugada syndrome in adults, but need further investigation in the younger age group.

In the case of a negative result, the presence of an abnormal electrical impulse generation or propagation provides invaluable confirmation of the clinical expression of the disease and helps guide the follow-up.

Because of scarce reports on the Brugada syndrome in the paediatric age, it is not possible to accurately define the value of programmed ventricular stimulation for risk stratification. No systematic studies have yet been performed to understand the mid- and 
long-term risks of a positive ventricular stimulation in young patients; further, the ideal age to start performing invasive risk stratification is unknown.

As shown by the study by Andorin et al, ${ }^{7}$ different centres seem to be using different protocols.

It has been our institutional practice to recommend risk stratification by means of ventricular stimulation in young patients with severe syncope and a diagnosis of the Brugada syndrome by means of spontaneous or drug-induced type I electrocardiography. In this age group, a patient-oriented management approach should be considered on an individual basis, taking into consideration the clinical circumstances and preferences of the family. We believe that by constructing a large and standardised database, we are building evidence that in the long term will help better understand this enigmatic disease and guide therapeutic decisions.

M. Cecilia Gonzalez Corcia Heart Rhythm Management Centre, UZ Brussels-VUB, Brussels, Belgium

\author{
Pediatric Cardiology Department, St Luc Hospital,
} Brussels, Belgium
Pedro Brugada

Heart Rhythm Management Centre, UZ Brussels-VUB,

Brussels, Belgium

\section{References}

1. Brugada P, Brugada J. Right bundle branch block, persistent ST segment elevation and sudden cardiac death: a distinct clinical and electrocardiographic syndrome. A multicenter report. J Am Coll Cardiol 1992; 20: 1391-1396.

2. Gonzalez Corcia MC, De Asmundis C, Chierchia GB, et al. Brugada syndrome in the paediatric population: a comprehensive approach to clinical manifestations, diagnosis, and management. Cardiol Young 2016; 26: 1044-1055.

3. Gonzalez Corcia MC, Sieira J, Sarkozy A, et al. Brugada syndrome in the young: an assessment of risk factors predicting future events. Europace, 2016. [Epub ahead of print] doi: 10.1093/europace/euw206.

4. Hermida JS, Lemoine JL, Aoun FB, Jarry G, Ray JL, Quiet JC. Prevalence of the Brugada syndrome in an apparently healthy population. Am J Cardiol 2000; 86: 91-94.

5. Priori S, Napolitano C, Giordano U, Collisani G, Memmi M. Brugada syndrome and sudden cardiac death in children. Lancet 2000; 355: 808-809.

6. Andorin A, Behr ER, Denjoy I, et al. The impact of clinical and genetic findings on the management of young patients with Brugada syndrome. Heart Rhythm 2016; 13: 1274-1282.

7. Priori SG, Wilde AA, Horie M, et al. HRS/EHRA/APHRS expert consensus statement on the diagnosis and management of patients with inherited primary arrhythmia syndromes. Heart Rhythm 2013; 10: 1932-1963. 